\title{
Stents Farmacológicos no Mundo Real: Impacto de sua Disponibilidade no Perfil de Pacientes Tratados por Meio de Intervenção Coronária Percutânea em um Hospital Público
}

\author{
Luiz Fernando Tanajura¹, Fausto Feres ${ }^{1}$, Alexandre C. Abizaid ${ }^{1}$, Luiz Alberto P. Mattos ${ }^{1}$, \\ Dimytri A. Siqueira1, Marinella P. Centemero', Áurea J. Chaves ${ }^{1}$, Andréa S. Abizaid ${ }^{1}$, \\ Amanda Guerra M. R. Sousa', J. Eduardo M. R. Sousa'
}

\section{RESUMO}

Introdução: Pacientes submetidos a intervenção percutânea em hospitais públicos brasileiros não têm acesso aos stents farmacológicos. No início de 2006, participamos de um registro multicêntrico internacional que disponibilizava o uso rotineiro dessas próteses, no cenário do mundo real. Neste artigo, procuramos identificar os perfis clínico, angiográfico e do procedimento de pacientes consecutivamente tratados em períodos subseqüentes de dois meses, de acordo com a disponibilidade desses modelos, visando à identificação de eventuais alterações nesses perfis. Método: Estudo observacional de uma série de 471 pacientes, divididos em dois grupos: A, 229 casos dilatados na vigência da disponibilidade de stents farmacológicos; e B, 242 pacientes subseqüentes tratados da forma usual. Não houve critérios de inclusão/exclusão. Resultados: Stents farmacológicos foram mais implantados no grupo A (44\% vs. $2 \%$; $p<0,0001)$. No que se refere às características de base, observou-se predomínio significante de diabéticos dependentes de insulina em A ( $8 \%$ vs. $3 \%$; $p=0,02)$, o mesmo ocorrendo com lesões-alvo tipos B2 ou C (73\% vs. 57\%; $p<0,0001)$, lesões situadas em bifurcações (15\% vs. $9 \%$; $p=0,02)$ e intervenções multiarteriais ( $15 \%$ vs. $6 \% ; p=0,003)$. A angiografia quantitativa identificou os casos de A como portadores de estenoses situadas em vasos de menor calibre $(2,4 \mathrm{~mm}$ vs. $2,6 \mathrm{~mm} ; \mathrm{p}=0,0004)$, também exibindo lesões mais longas $(14,9 \mathrm{~mm}$ vs. $12,7 \mathrm{~mm} ; \mathrm{p}=0,0008)$. Conclusões: A disponibilidade dos stents farmacológicos gerou alterações no perfil dos casos tratados, que passou a abordar situações mais predispostas à reestenose, como os diabéticos dependentes de insulina, os multiarteriais com lesões de alta complexidade e os portadores de lesões mais longas em vasos de fino calibre.

DESCRITORES: Coronariopatia. Contenedores. Angioplastia transluminal percutânea coronária.

\begin{abstract}
SUMMARY
Drug-Eluting Stents in the Real World Scenario: the Impact of Their Availability in the Profile of Patients Treated Percutaneously in a Public Hospital

Introduction: Patients submitted to percutaneous intervention in public hospitals in Brazil have no access to DES. At the beginning of 2006, we participated in an international multicenter registry which made available the routine use of these prosthesis, in a "real world" scenario. In this article, we intend to identify the clinical, angiographical and procedural profiles of the patients, consecutively treated in twomonth subsequent periods, according to the availability of these models, with the aim of identifying eventual changes in their profiles. Method: Observational series of 471 patients, divided into two groups: A) 229 cases, treated during a period of availability of DES for routine use; and B) 242 patients subsequently treated as usual (no routine avalilability of DES). There were no inclusion/exclusion criteria. Results: More DES were implanted in group A ( $44 \%$ vs. $2 \%$; $p<0.0001)$. Regarding baseline clinical and angiographic characteristics, a significant predominance of insulin-dependent diabetics was observed in group A ( $8 \%$ vs. $3 \% ; p=0.02)$, as well as $\mathrm{B} 2$ or $\mathrm{C}$ lesions ( $73 \%$ vs. $57 \%$; $\mathrm{p}<0.0001)$; lesions in bifurcations $(15 \%$ vs. $9 \% ; p=0.02)$, and multiarterial interventions $(15 \%$ vs. $6 \% ; p=0.003)$. Quantitative angiography identified the A group cases as bearers of stenosis placed in smaller vessels $(2.4 \mathrm{~mm}$ vs. $2.6 \mathrm{~mm} ; \mathrm{p}=0.0004)$, also exhibiting longer lesions (14.9 $\mathrm{mm}$ vs. $12.7 \mathrm{~mm} ; \mathrm{p}=0.0008$ ). Conclusions: The availability of DES changed the profile of the patients treated, being more likely situations prone to restenosis, such as insulin dependent diabetics, multiarterial disease, patients presenting complex lesions, longer lesions and smaller target vessels.
\end{abstract}

DESCRIPTORS: Coronary disease. Stents. Angioplasty, transluminal, percutaneous coronary.

\footnotetext{
Instituto Dante Pazzanese de Cardiologia - São Paulo, SP. Correspondência: Luiz Fernando Tanajura. Av. Dr. Dante Pazzanese, 500 - Vila Mariana - São Paulo, SP - CEP 04012-909 Tel.: (11) 5085-6000 • E-mail: Iftanajura@uol.com.br Recebido em: 7/1/2008 • Aceito em: 6/2/2008
} 
Tanajura LF, et al. Stents Farmacológicos no Mundo Real: Impacto de sua Disponibilidade no Perfil de Pacientes Tratados por Meio de Intervenção Coronária Percutânea em um Hospital Público. Rev Bras Cardiol Invas. 2008;16(1):31-36.

A introdução e o posterior desenvolvimento dos stents farmacológicos, dispositivos associados a reduções substanciais das cifras de reestenose tanto angiográfica como clínica, representaram verdadeira revolução no âmbito do tratamento das lesões coronárias por meio de intervenção coronária percutânea (ICP), possibilitando a ampliação das indicações dessa opção terapêutica para cenários nos quais ela não seria cogitada na era dos stents não-revestidos, pela elevada possibilidade de recidiva ${ }^{1-5}$. Temores quanto à segurança desses stents, mais especificamente relacionados à ocorrência de trombose tardia, mostraramse infundados ${ }^{6-8}$.

No entanto, em nosso meio, a maior parte dos casos não tem acesso a esse tipo de prótese, em razão de a Previdência Social ainda não cobrir o custo de sua aquisição. Recentemente, o Instituto Dante Pazzanese de Cardiologia participou de um registro internacional (E-FIVE), que possibilitou a ampla utilização desses modelos de forma rotineira. Os pacientes que se comprometessem a participar, fazendo o acompanhamento médico por dois anos nesse hospital, poderiam ser tratados com stents farmacológicos, sem restrição de número.

Essa investigação visou ao esclarecimento da influência da disponibilidade do uso rotineiro desses instrumentos na seleção de casos, mais precisamente nas características clínicas, angiográficas e relacionadas ao procedimento percutâneo, de uma série consecutiva de pacientes submetidos a ICP em um hospital terciário de grande movimento, no cenário do mundo real.

\section{MÉTODO}

O registro E-FIVE permitiu o recrutamento de todo e qualquer paciente tratado por meio de ICP nos hospitais envolvidos, desde que: 1) pelo menos um stent Endeavor $^{\circledR}$ (Medtronic, Estados Unidos), com liberação de zotarolimus (endoprótese de segunda geração com segurança e efetividade comprovadas em ensaios clínicos prévios ${ }^{9}$ ), pudesse ser implantado para o tratamento de estenose significante $(>50 \%$ ), em presença de isquemia espontânea (angina estável ou síndromes coronárias agudas com ou sem elevação do segmento ST) ou induzida por testes funcionais; 2) o paciente se dispusesse a fazer o acompanhamento clínico como já mencionado. As exclusões constituíram-se essencialmente de gestantes e casos com restrições ao uso do esquema antiplaquetário duplo pelo período indicado. O objetivo primário do ensaio foi avaliar o porcentual de eventos cardíacos maiores aos dois anos de evolução.

Dessa forma, procedeu-se à análise retrospectiva de todos os pacientes dilatados em nosso Serviço, entre 6/1/2006 e 6/3/2006, período de recrutamento do E-FIVE (grupo A: 229 pacientes tratados, dos quais 100 foram incluídos no E-FIVE), cujas características clínicas e angiográficas de base, bem como os dados relacionados ao procedimento, foram comparados aos dos 242 casos (grupo B) consecutivamente tratados nos dois meses subseqüentes (período compreendido entre 7/3/2006 e 7/5/2006).

Os pacientes foram identificados a partir de um banco de dados informatizado, no qual foram incluídos de forma prospectiva. A natureza do estudo foi observacional.

As ICP com implante de endopróteses coronárias foram realizadas pela técnica convencional de liberação ótima, com implante direto ou pré-dilatação, o que era deixado a critério do cardiologista intervencionista.

O esquema farmacológico adjunto consistiu de: 1) heparina não-fracionada, na dose de $100 \mathrm{UI} / \mathrm{kg}$ de peso, administrada imediatamente antes do procedimento com o objetivo de atingir tempo de coagulação ativado de 250 segundos ou mais; 2) aspirina (100 $\mathrm{mg} / \mathrm{dia}$ ), iniciada pelo menos na véspera da intervenção e mantida indefinidamente; 3) clopidogrel (dose de ataque de $300 \mathrm{mg}$, seguida por $75 \mathrm{mg} / \mathrm{dia}$ ) ou ticlopidina (500 mg/dia), ambas com pré-tratamento. O protocolo do registro E-FIVE estipulou o emprego do esquema antiplaquetário duplo por pelo menos 12 semanas; após esse período, sua manutenção ficaria a critério da equipe médica. Nos casos tratados por meio de modelos não-revestidos, a associação dos antiplaquetários foi mantida por 30 dias. Inibidores da glicoproteína Ilb/IIla foram utilizados nas doses habituais, quando indicados.

Em relação à análise da angiografia coronária préprocedimento, foram consideradas significativas lesões com diâmetro de estenose superior a 50\%. As lesõesalvo foram classificadas de acordo com a proposta da American College of Cardiology/American Heart Association Task Force ${ }^{10}$. A função ventricular esquerda foi avaliada globalmente pela análise da fração de ejeção do ventrículo esquerdo. A angiografia coronária quantitativa não foi realizada de forma rotineira, o mesmo acontecendo com o ultra-som intracoronário.

Definições: 1) considerou-se sucesso primário a obtenção de estenose residual inferior a 50\%, na ausência de complicações maiores (óbito, infarto do miocárdio ou cirurgia de revascularização em caráter de urgência) na fase hospitalar; 2) infarto do miocárdio foi caracterizado pela presença de novas ondas Q no eletrocardiograma de 12 derivações e/ou por elevações da creatina quinase fração $\mathrm{MB}(\mathrm{CK}-\mathrm{MB})$ superiores a três vezes o valor normal; 3) foram considerados eventos cardíacos maiores óbito de causa cardiovascular, infarto e revascularização miocárdica durante a evolução clínica tardia.

A comparação entre as variáveis quantitativas foi feita utilizando-se o teste $t$ de Student. Para as comparações referentes às variáveis qualitativas, utilizou-se o teste estatístico do qui-quadrado. Consideraram-se significantes valores de $p<0,05$. 
Tanajura LF, et al. Stents Farmacológicos no Mundo Real: Impacto de sua Disponibilidade no Perfil de Pacientes Tratados por Meio de Intervenção Coronária Percutânea em um Hospital Público. Rev Bras Cardiol Invas. 2008;16(1):31-36.

\section{RESULTADOS}

A Tabela 1 apresenta as principais características clínicas de base, que foram igualmente distribuídas entre os grupos. No entanto, entre os diabéticos, os casos dependentes do uso de insulina foram significativamente mais observados no grupo A ( $8 \%$ vs. $3 \%$; $p=0,02)$. Também não foram observadas diferenças no que se refere aos antecedentes de revascularização miocárdica prévia (percutânea ou cirúrgica).

Em relação à terapêutica clínica adjunta, os inibidores da glicoproteína Ilb/Illa foram administrados de forma esporádica em ambos os grupos (2\%).

A função ventricular esquerda era preservada (fração de ejeção igual ou superior a 55\%) na maioria dos pacientes $(66 \%$ vs. $63 \%$; $p=N S)$, o mesmo ocorrendo em relação à presença de doença multiarterial na angiografia coronária (54\% vs. 49\%; p = NS). Angiografia coronária quantitativa foi significativamente mais realizada nos casos do grupo A (94\% vs. $80 \%$; $\mathrm{p}<0,0001)$. As demais características observadas na angiografia diagnóstica estão expressas na Tabela 2.

No tocante aos dados relacionados com o procedimento, a revascularização completa foi semelhante nos dois grupos (57\% vs. 54\%; p = NS). Intervenções para o tratamento de múltiplos vasos predominaram no grupo A ( $15 \%$ vs. $6 \% ; p=0,003)$, assim como o uso concomitante do ultra-som intracoronário $(30 \%$ vs. $10 \% ; p<0,0001)$ e o implante de stents com diâmetro igual ou menor que 2,5 mm (20\% vs. $14 \%$; $p=0,04)$. Stents farmacológicos foram implantados em $44 \%$ dos casos do grupo A e em $2 \%$ dos pacientes do grupo B ( $p<0,0001)$. Modelos com extensão igual ou superior a $30 \mathrm{~mm}$ foram implantados em 10\% dos pacientes dos dois grupos.
Sucesso do procedimento ocorreu em 98\% dos casos de ambos os grupos.

\section{DISCUSSÃo}

A utilização de stents farmacológicos acompanhouse de drásticas reduções dos porcentuais de reestenose angiográfica e revascularizações adicionais no primeiro ano após a intervenção percutânea, o que é observado em praticamente todos os casos tratados por meio desses dispositivos, independentemente do modelo empregado ${ }^{1-5,9}$, o que causou extrapolação das indicações dessas próteses para variedades anatômicas ainda não devidamente avaliadas por meio de ensaios clínicos específicos ${ }^{11-14}$. Mais recentemente, registros envolvendo casos dilatados no mundo real, ou seja, sem critérios específicos de inclusão/exclusão, demonstraram inclusive redução tardia da mortalidade nos que utilizaram stents farmacológicos, comparativamente ao uso das próteses não-revestidas, ratificando sua efetividade e segurança ${ }^{11,15}$. As possíveis explicações para esse resultado são: redução das complicações decorrentes de reintervenções em casos de recidiva, maior índice de revascularização miocárdica completa funcional, e maior alívio da isquemia crônica ${ }^{15}$.

Em nosso meio, os pacientes tratados em hospitais públicos de grande movimento, como é o caso de nosso Serviço, não têm acesso a essa tecnologia, pelo fato de o Sistema Único de Saúde, fonte predominante de custeio dos procedimentos efetuados, não autorizar o implante desses dispositivos, em razão do custo ainda elevado. Pela elevada perspectiva de reestenose, muitos casos deixam de ser tratados nas situações de indisponibilidade dessas próteses, embora, do ponto de vista anatômico, pudessem ser tratados por via percutânea. Observou-se que quase metade dos pacien-

TABELA 1

Características clínicas de base

\begin{tabular}{lccc}
\hline Variáveis & Grupo A $(\mathbf{n}=\mathbf{2 2 9})$ & Grupo B $(\mathbf{n}=\mathbf{2 4 2})$ & $\mathbf{p}$ \\
\hline Idade (média) & 61,1 anos & 61,7 anos & NS \\
Sexo masculino & $159(69 \%)$ & $171(71 \%)$ & NS \\
Tabagismo (atual ou não) & $119(52 \%)$ & $135(56 \%)$ & NS \\
Diabetes melito & $71(31 \%)$ & $72(32 \%)$ & NS \\
HAS & $189(83 \%)$ & $205(85 \%)$ & NS \\
Hipercolesterolemia & $162(71 \%)$ & $179(74 \%)$ & NS \\
Infarto prévio & $99(43 \%)$ & $116(47 \%)$ & NS \\
Apresentação clínica & $91(40 \%)$ & $78(32 \%)$ & \\
$\quad$ Angina estável & $35(15 \%)$ & $34(14 \%)$ & $106(44 \%)$ \\
$\quad$ Angina instável & $85(37 \%)$ & $24(10 \%)$ & \\
IAM recente & $18(8 \%)$ & & \\
Isquemia silenciosa & & \\
\hline
\end{tabular}

$\mathrm{n}$ = número de pacientes; HAS = hipertensão arterial sistêmica; IAM = infarto do miocárdio; NS = não significativa. 
Tanajura LF, et al. Stents Farmacológicos no Mundo Real: Impacto de sua Disponibilidade no Perfil de Pacientes Tratados por Meio de Intervenção Coronária Percutânea em um Hospital Público. Rev Bras Cardiol Invas. 2008;16(1):31-36.

TABELA 2

Resultados da angiografia coronária pré-procedimento

\begin{tabular}{|c|c|c|c|}
\hline Variáveis & $\begin{array}{c}\text { Grupo A } \\
(n=229) \\
\text { média (DP) }\end{array}$ & $\begin{array}{c}\text { Grupo B } \\
(n=242) \\
\text { média (DP) }\end{array}$ & $\mathbf{p}$ \\
\hline Artéria tratada & & & NS \\
\hline Descendente anterior & $38 \%$ & $42 \%$ & \\
\hline Coronária direita & $32 \%$ & $30 \%$ & \\
\hline Circunflexa & $26 \%$ & $25 \%$ & \\
\hline Enxertos venosos & $4 \%$ & $3 \%$ & \\
\hline Tipo de lesão & & & $<0,0001$ \\
\hline $\mathrm{A} / \mathrm{B}_{1}$ & $27 \%$ & $43 \%$ & \\
\hline $\mathrm{B}_{2} / \mathrm{C}$ & $73 \%$ & $57 \%$ & \\
\hline Diâmetro de referência $(\mathrm{mm})$ & $2,4(0,7)$ & $2,6(0,8)$ & 0,0004 \\
\hline DML pré-implante (mm) & $0,7(0,8)$ & $0,7(0,4)$ & NS \\
\hline Extensão da lesão-alvo (mm) & $14,9(11)$ & $12,7(8,3)$ & 0,008 \\
\hline Lesões reestenóticas & $5 \%$ & $3 \%$ & NS \\
\hline Lesões em bifurcações & $15 \%$ & $9 \%$ & 0,02 \\
\hline Localização distal & $12 \%$ & $5 \%$ & 0,005 \\
\hline
\end{tabular}

$\mathrm{n}=$ número de pacientes; DP = desvio padrão; DML = diâmetro mínimo da luz; NS = não significativa.

tes de nossa casuística (44\%) foi dilatada utilizando stents farmacológicos, números similares aos de registros europeus contemporâneos ${ }^{16,17}$, porém inferiores aos de registros norte-americanos ${ }^{12}$.

Os resultados desta investigação confirmam essa assertiva. Avaliando-se coortes distintas de pacientes cujo tratamento foi separado por um período de tempo muito curto (dois meses), demonstrou-se que a disponibilidade do emprego de stents farmacológicos foi acompanhada de clara alteração do perfil dos casos dilatados, que passou a incluir mais freqüentemente situações com alta predisposição para o desenvolvimento de reestenose, tais como os diabéticos dependentes de insulina, os portadores de lesões de alta complexidade, os que exibiam estenoses em vasos de fino calibre, muitas localizadas nas porções distais das artérias, e as bifurcações. Igualmente relevante foi a possibilidade de propiciar aumento significante nas ICP de múltiplos vasos, que, todavia, não influenciou a obtenção de maiores índices de revascularização completa.

O diabetes melito sempre foi considerado um preditor independente de reestenose, o que é ainda mais exacerbado nas situações de pacientes dependentes de insulina, em razão da resposta vascular hiperproliferativa decorrente da injúria causada pela $\mathrm{ICP}^{11,18}$. Adicionalmente, essa doença se acompanha de acometimento das porções mais distais dos vasos, áreas, em geral, de menor diâmetro, o que limita os resultados da revascularização miocárdica por qualquer método ${ }^{11,18}$. Investigações randomizadas já comprovaram a grande vantagem dos stents farmacológicos nos diabéticos, com reduções dramáticas das revascularizações decorrentes de reestenose, o que torna lógico o achado da presente investigação, que abrangeu um cenário sem seleção prévia de $\operatorname{casos}^{11,19}$.

O mesmo ocorre naqueles que evidenciam acometimento de vasos de menor diâmetro de referência, também associados a maiores taxas de reobstrução pelo critério binário ${ }^{11,14,20,21}$. Aparentemente, essa predisposição deve-se ao fato de os volumes de hiperplasia intimal não dependerem do diâmetro da artéria-alvo, facilitando o desenvolvimento de reestenose nesses vasos $^{20,21}$. O benefício do implante de stents farmacológicos nesses casos também já está claramente demonstrado, igualmente explicando o achado dessa investigação ${ }^{20,21}$. O predomínio de lesões de localização distal no grupo A também nos pareceu estar associado a esse achado anatômico.

$\mathrm{Na}$ literatura, observações menos robustas foram registradas no tocante às lesões em bifurcações ${ }^{11,14}$, nas quais a grande dúvida atual é relacionada à técnica de implante $^{22}$, e não ao uso dos modelos com liberação de fármacos, e às estenoses mais longas, cuja magnitude do benefício parece ser mais evidente quanto mais extensa for a lesão-alvo, conforme relatos recentes ${ }^{11,14,23}$.

É nesse conjunto de situações adversas, tornando as lesões-alvo de muito alta complexidade e muito mais propensas à reestenose (Figura 1), que o benefício com o uso de stents farmacológicos ${ }^{11-14}$ se mostra mais efetivo, quando comparado aos stents não-revestidos, fato comprovado pelo aumento significante do porcentual de ICP de múltiplos vasos quando esses 
Tanajura LF, et al. Stents Farmacológicos no Mundo Real: Impacto de sua Disponibilidade no Perfil de Pacientes Tratados por Meio de Intervenção Coronária Percutânea em um Hospital Público. Rev Bras Cardiol Invas. 2008;16(1):31-36.

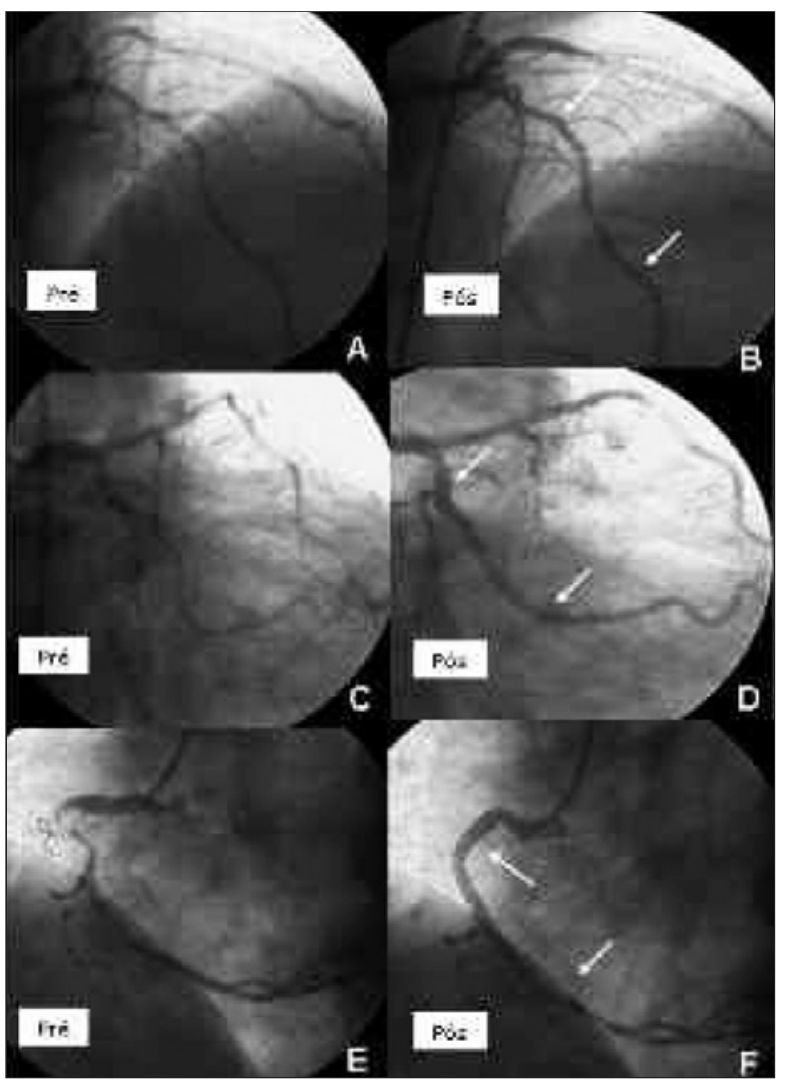

Figura 1 - Paciente diabético com doença coronária triarterial (A C e E), encaminhado ao Instituto Dante Pazzanese de Cardiologia para revascularização cirúrgica do miocárdio. A disponibilidade de stents com liberação de medicamentos possibilitou seu tratamento por via percutânea, utilizando seis endopróteses. As intervenções realizadas nas artérias descendente anterior (B), circunflexa (D) e coronária direita (F) necessitaram do implante de múltiplas endopróteses para o tratamento de lesões segmentares.

modelos estavam disponibilizados. Entretanto, embora não seja o objetivo desta investigação, deve-se salientar também que essas indicações se acompanham de maior risco de complicações maiores imediatas e tardias, como demonstrado pelos resultados do registro EVENT ${ }^{13}$, no qual a ICP em situações de indicação ainda não precisamente comprovada por meio de ensaios clínicos específicos (off-label) acompanhou-se de aumentos significativos dos eventos cardíacos maiores na fase hospitalar $(11 \%$ vs. $5 \%$; p < 0,001) e na evolução clínica de um ano $(17 \%$ vs. $9 \%$; $p<0,001)$. Em futuro próximo, os resultados de estudos clínicos normativos que já estão em andamento, a maioria dos quais compara ICP com stents farmacológicos e cirurgia de revascularização em presença de doença coronária multiarterial de alta complexidade, demonstrarão de forma mais precisa qual é a melhor opção terapêutica para esses casos.

Outro detalhe que merece destaque foi o maior porcentual de pacientes do grupo A avaliados por meio do ultra-som intracoronário. Sem dúvida, o maior responsável por esse fato foi o uso de stents farmacológicos, não apenas por esse exame tornar os implantes mais precisos, o que muitas vezes é essencial quando esses modelos são liberados, mas também pela possibilidade de comparar os resultados imediatos com os tardios, pela aferição, entre outros dados, do volume de hiperplasia neointimal, cerne da reestenose intrastent, em circunstâncias de mundo real, o que torna os resultados mais fidedignos que os observados no cenário restrito dos estudos randomizados ${ }^{11,14,24,25}$.

\section{Limitações}

As seguintes restrições devem ser aventadas: 1) a análise foi feita de forma retrospectiva; 2) a angiografia coronária quantitativa foi realizada mais freqüentemente nos casos do grupo A (se houvesse igual proporção de pacientes analisados em ambos os grupos, algumas das diferenças aferidas nesse quesito poderiam não ser observadas).

\section{CONCLUSÕES}

A disponibilidade da utilização de stents farmacológicos proporcionou uma mudança no perfil dos pacientes tratados, possibilitando a abordagem de casos mais propensos ao desenvolvimento da reestenose: diabéticos dependentes de insulina, intervenções multiarteriais envolvendo lesões-alvo de alta complexidade, vasos de fino calibre com estenoses na porção distal e bifurcações. Podemos afirmar, sem receio, que muitos dos casos em questão teriam sido recusados para dilatação em situações nas quais esses instrumentos não estivessem disponíveis.

\section{REFERÊNCIAS BIBLIOGRÁFICAS}

1. Sousa JE, Costa MA, Abizaid A, Abizaid AS, Feres F, Pinto $\mathrm{IM}$, et al. Lack of neointimal proliferation after implantation of sirolimus-coated stents in human coronary arteries: a quantitative coronary angiography and three-dimensional intravascular ultrasound study. Circulation. 2001;103(2):192-5.

2. Morice MC, Serruys PW, Sousa JE, Fajadet J, Ban Hayashi $\mathrm{E}$, Perin $\mathrm{M}$, et al. A randomized comparison of a sirolimuseluting stent with a standard stent for coronary revascularization. N Engl J Med. 2002;346(23):1773-80.

3. Stone GW, Ellis SG, Cox DA, Hermiller J, O'Shaughnessy C, Mann JT, et al. A polymer-based, paclitaxel-eluting stent in patients with coronary artery disease. N Engl J Med. 2004;350(3):221-31.

4. Ong AT, Serruys PW, Aoki J, Hoye A, van Mieghem CA, Rodriguez-Granillo GA, et al. The unrestricted use of paclitaxelversus sirolimus-eluting stents for coronary artery disease in an unselected population: one-year results of the TaxusStent Evaluated at Rotterdam Cardiology Hospital (T-SEARCH) registry. J Am Coll Cardiol. 2005;45(7):1135-41.

5. Lemos PA, Hoye A, Goedhart D, Arampatzis CA, Saia F, van der Giessen WJ, et al. Clinical, angiographic, and procedural predictors of angiographic restenosis after sirolimuseluting stent implantation in complex patients: an evaluation from the Rapamycin-Eluting Stent Evaluated At Rotterdam Cardiology Hospital (RESEARCH) study. Circulation. 2004;109(11):1366-70. 
Tanajura LF, et al. Stents Farmacológicos no Mundo Real: Impacto de sua Disponibilidade no Perfil de Pacientes Tratados por Meio de Intervenção Coronária Percutânea em um Hospital Público. Rev Bras Cardiol Invas. 2008;16(1):31-36.

6. Stone GW, Moses JW, Ellis SG, Schofer J, Dawkins KD Morice MC, et al. Safety and efficacy of sirolimus- and paclitaxel-eluting coronary stents. N Engl J Med. 2007;356(10): 998-1008.

7. Mauri L, Hsieh WH, Massaro JM, Ho KK, D'Agostino R, Cutlip DE. Stent thrombosis in randomized clinical trials of drug-eluting stents. N Engl J Med. 2007;356(10):1020-9.

8. Spaulding C, Daemen J, Boersma E, Cutlip DE, Serruys PW. A pooled analysis of data comparing sirolimus-eluting stents with bare-metal stents. N Engl J Med. 2007;356(10):989-97.

9. Fajadet J, Wijns W, Laarman GJ, Kuck KH, Ormiston J, Münzel $\mathrm{T}$, et al. Randomized, double-blind, multicenter study of the Endeavor zotarolimus-eluting phosphorylcholineencapsulated stent for treatment of native coronary artery lesions: clinical and angiographic results of the ENDEAVOR II trial. Circulation. 2006;114(8):798-806.

10. Smith SC Jr, Dove JT, Jacobs AK, Kennedy JW, Kereiakes D, Kern MJ, et al. ACC/AHA guidelines for percutaneous COronary intervention (revision of the 1993 PTCA guidelines) executive summary: a report of the American College of Cardiology/American Heart Association task force on practice guidelines (Committee to revise the 1993 guidelines for percutaneous transluminal coronary angioplasty) endorsed by the Society for Cardiac Angiography and Interventions. Circulation. 2001;103(24):3019-41.

11. Serruys PW, Kutryk MJ, Ong AT. Coronary-artery stents. N Engl J Med. 2006;354(5):483-95.

12. Beohar N, Davidson CJ, Kip KE, Goodreau L, Vlachos HA Meyers SN, et al. Outcomes and complications associated with off-label and untested use of drug- eluting stents. JAMA. 2007;297(18):1992-2000.

13. Win HK, Caldera AE, Maresh K, Lopez J, Rihal CS, Parikh $M A$, et al. Clinical outcomes and stent thrombosis following off-label use of drug-eluting stents. JAMA. 2007;297(18): 2001-9.

14. Colombo A, Chieffo A. Drug-eluting stent update 2007: part III: Technique and unapproved/unsettled indications (left main, bifurcations, chronic total occlusions, small vessels and long lesions, saphenous vein grafts, acute myocardial infarctions, and multivessel disease). Circulation. 2007;116 (12):1424-32.

15. Tu JV, Bowen J, Chiu M, Ko DT, Austin PC, He Y, et al Effectiveness and safety of drug-eluting stents in Ontario. $N$ Engl J Med. 2007;357(14):1393-402.
16. Lagerqvist B, James SK, Stenestrand U, Lindbäck J, Nilsson T, Wallentin L. Long-term outcomes with drug-eluting stents versus bare-metal stents in Sweden. N Engl J Med. 2007;356 (10):1009-19.

17. Marzocchi A, Saia F, Piovaccari G, Manari A, Aurier E, Benassi A, et al. Long-term safety and efficacy of drugeluting stents: two-year results of the REAL (REgistro Regionale AngiopLastiche dell'Emilia Romagna) multicenter registry. Circulation. 2007;115(25):3181-8.

18. Yang TH, Park SW, Hong MK, Park DW, Park KM, Kim YH, et al. Impact of diabetes mellitus on angiographic and clinical outcomes in the drug-eluting stents era. Am J Cardiol. 2005;96(10):1389-92.

19. Sabaté $M$, Jiménez-Quevedo P, Angiolillo DJ, Gómez-Hospital JA, Alfonso F, Hernández-Antolín R, et al Randomized comparison of sirolimus-eluting stent versus standard stent for percutaneous coronary revascularization in diabetic patients: the diabetes and sirolimus-eluting stent (DIABETES) trial. Circulation. 2005;112(14):2175-83.

20. Ardissino D, Cavallini C, Bramucci E, Indolfi C, Marzocchi A, Manari A, et al. Sirolimus-eluting vs uncoated stents for prevention of restenosis in small coronary arteries: a randomized trial. JAMA. 2004;292(22):2727-34.

21. Meier B, Sousa E, Guagliumi G, Van den Branden F, Grena$\operatorname{dier}$ E, Windecker S, et al. Sirolimus-eluting coronary stents in small vessels. Am Heart J. 2006;151(5):1019.e1-7.

22. Steigen TK, Maeng M, Wiseth R, Erglis A, Kumsars I, Narbute I, et al. Randomized study on simple versus complex stenting of coronary artery bifurcation lesions: the Nordic bifurcation study. Circulation. 2006;114(18):1955-61.

23. Kim YH, Park SW, Lee SW, Park DW, Yun SC, Lee CW, et al. Sirolimus-eluting stent versus paclitaxel-eluting stent for patients with long coronary artery disease. Circulation. 2006;114(20):2148-53.

24. Tanajura LFL. Comparação randomizada entre o implante de stents com ou sem carbeto de silício amorfo para a prevenção da reestenose coronária [Tese de Doutorado]. São Paulo: Universidade de São Paulo, Faculdade de Medicina; 2003. 122p.

25. Hong MK, Mintz GS, Lee CW, Park DW, Park KM, Lee BK, et al. Late stent malapposition after drug-eluting stent implantation: an intravascular ultrasound analysis with longterm follow-up. Circulation. 2006;113(3):414-9. 